\title{
Sensitive Detection of Biomolecules by Surface Enhanced Raman Scattering using Plant Leaves as Natural Substrates
}

\author{
Vipul Sharma and Venkata Krishnan* \\ School of Basic Sciences and Advanced Materials Research Center, Indian Institute of Technology Mandi, Kamand, Mandi 175005, \\ H.P., India
}

\begin{abstract}
Detection of biomolecules is highly important for biomedical and other biological applications. Although several methods exist for the detection of biomolecules, surface enhanced Raman scattering (SERS) has a unique role in greatly enhancing the sensitivity. In this work, we have demonstrated the use of natural plant leaves as facile, low cost and eco-friendly SERS substrates for the sensitive detection of biomolecules. Specifically, we have investigated the influence of surface topography of five different plant leaf based substrates, deposited with $\mathrm{Au}$, on the SERS performance by using L-cysteine as a model biomolecule. In addition, we have also compared the effect of sputter deposition of Au thin film with dropcast deposition of $\mathrm{Au}$ nanoparticles on the leaf substrates. Our results indicate that L-cysteine could be detected with high sensitivity using these plant leaf based substrates and the leaf possessing hierarchical micro/nanostructures on its surface shows higher SERS enhancement compared to a leaf having a nearplanar surface. Furthermore, leaves with drop-casted Au nanoparticle clusters performed better than the leaves sputter deposited with a thin Au film.
\end{abstract}

\section{Introduction}

There are various types of important biomolecules that are responsible for the maintenance of metabolic processes in living organisms [1]. In the scientific field, the detection of these biomolecules is essential in various analytical, medical, biochemical and pharmaceutical applications $[2,3]$. Biomolecules are generally composed of organic compounds, which include large macromolecules, like proteins, carbohydrates, lipid $\mathrm{s}$, and nucleic acids, as well as small molecules such as various metabolites and natural products [3]. In general, the most common techniques used to detect and study these biomolecules include polymerase chain reaction (PCR) and enzyme linked immunsorbent assay (ELISA) [4]. The other methods to the study the biomolecules include various microscopic and spectroscopic techniques, such as fluorescence microscopy, Fourier transform infrared spectroscopy, UV-vis absorption spectroscopy and Raman spectroscopy [5]. All the methods of detection and analysis of biomolecules are unique and give valuable insights about the structure and composition of biomolecules. Amino acids are considered as the basic building blocks that combine together to form proteins. L-cysteine, is reported as a 'non-essential' amino acid that is typically synthesized by the human body and is responsible for the stabilization of secondary structure of proteins [6]. Since this molecule plays an important role in deactivating free radicals (antioxidant), it has been the focus of several studies [7].

Raman spectroscopy is a valuable investigation tool that employs distinctive vibrational patterns for the detection of molecules [8]. It has direct application in the chemical and bio sensing, however its use is very much limited due to low intensity of normal Raman scattering signals [9]. Nevertheless, much more sensitive Raman techniques are being developed such as, surface enhanced Raman scattering (SERS), wherein the method provides valuable information on the molecules and their local interactions than normal Raman spectroscopy by leveraging the characteristic and exclusive properties of plasmonic nanoparticles leading to enhanced detection limits while maintaining the very good sensitivity [10]. When the electromagnetic radiation interacts with the oscillating cloud of conduction electrons of plasmonic nanoparticles, it leads to the electromagnetic energy confinement around the nanoparticles. Significant Raman signal amplification can be achieved by the introduction of a Raman active molecule in close proximity to the nanoparticles, and within the confined electromagnetic field [10,11]. The Raman scattering from an analyte adsorbed on the nanostructured metal surface can significantly increase in comparison to solution. The phenomenon was first observed by Fleischman in 1974 and elaborated by Van Duyne in 1977 [12]. The nanostructures of noble and transition metals onto which the molecules are adsorbed can lead to $10^{10}$ to $10^{15}$ fold enhancement of the intensity of Raman scattered light and the effect is reported to be strongest on $\mathrm{Ag}$ followed by $\mathrm{Au}$ and $\mathrm{Cu}[10]$.

Further moving in this field, improvement in the sensitivity of SERS signal and performance has been amplified by arranging the nanostructures into a threedimensional structure by many ways such as placing the plasmonic nanostructures directly or combining plasmonic nanostructures with non-plasmonic frameworks [13]. The preparation of 3D SERS substrates by merging plasmonic nanostructures with a roughened or grooved substrate is generally done by decorating noble metal nanostructures on specifically designed 3D frameworks [14]. In some of the previously reported

Corresponding author: vkn $@$,iitmandi.ac.in

(C) The Authors, published by EDP Sciences. This is an open access article distributed under the terms of the Creative Commons Attribution License 4.0 (http://creativecommons.org/licenses/by/4.0/). 
works, 3D SERS substrates have been produced by decoration of noble metal nanoparticles on various 3D templates $[15,16]$. For the creation of 3D nanostructured templates, methods that allow ordered hierarchy in the structure, like Langmuir-Blodgett technique [17], electrochemical methods [18], and lithography methods [19] are normally employed. The fabricated substrates exhibit high density of the metal nanostructures ordered in clusters. These interact with analytes and abundant nanometer level gaps to dramatically enhance the local electromagnetic fields upon excitation by incident laser. In the recent years, biodegradable substrates, such as biological fibers have been used to create 3D templates for decoration of plasmonic nanostructures [20]. There are also some reports where paper made up of cellulose fibers has been employed to create the 3D templates for the SERS applications. Very good SERS activity is reported for traditional SERS supports and many engineered substrates with improved designs, but they do have some limitations as it is very difficult to assemble the nanoparticles in a well-ordered and controllable manner for efficient and sensitive SERS detection [21].

Plasmonic metal decorated natural and bioinspired surfaces have already gained attention for SERS applications, given their good plasmonic properties and 3D hierarchical structure for the fabrication of the efficient SERS substrates [22]. There are natural surfaces present in different flora and fauna, which possess vibrant hierarchical structures, wettability and adhesion related properties [22]. For SERS applications, there are reports where researchers have used butterfly wings as a substrate, which provided very good enhancement factors and very low detection limits as well [23]. Recent reports include rose petals and Taro leaf which were directly used as 3D templates for SERS where the nanoparticle clusters were very nicely arranged over the microstructures present on the surfaces and provided very good SERS activity [24]. In one of our recent works, we have reported on the use of gold deposited plant leaves for SERS application for the detection of polar and non-polar analytes, wherein we had elaborated the role of surface morphology, wettability and deposition technique in determining the enhancement factor and detection limit [25]. The performance of the SERS substrates is generally dependent on two factors: the concentration of analytes and the existence of strong electromagnetic field $[15,26]$. Usually the Raman signal enhancement is dependent on the surface plasmons present over the nanoparticle surfaces and this enhancement is even more pronounced when two or more nanoparticles are in close proximity with each other creating the so called, hot spots. The number of these hot spots increases when the nanoparticles are present in clusters. Plasmonic hot spots can also be created on the $3 \mathrm{D}$ structures by deposition of thin films over them by taking advantage of the 3D hierarchy of the substrates [27].

In this work, we demonstrate the use of natural plant leaves as facile, low cost and eco-friendly SERS substrates for the sensitive detection of biomolecules, using L-cysteine as a representative molecule. Five different plant leaves possessing different 3D micro/ nanostructures on their surface have been examined to investigate the effect of topography on the intensity of SERS signals. Two different fabrication techniques were used to fabricate the plant leaf based SERS substrates. In one approach, a thin layer of Au was sputter coated on to the surface of the leaves, while in the other approach $\mathrm{Au}$ nanoparticles were drop casted on to the surface of the leaves. A comparison between the two fabrication approaches is also presented in this work.

\section{Experimental}

\subsection{Materials}

For the synthesis of $\mathrm{Au}$ nanoparticles, chloroauric acid $\left(\mathrm{HAuCl}_{4}\right)$, sodium citrate and hydroquinone were purchased from Sigma Aldrich. The fixation of fresh leaves was done using glutaraldehyde, potassium dihydrogen orthophosphate, di-sodium hydrogen orthophosphate and glycerol, all of which were supplied by Sigma Aldrich. The silicon wafer $(0.5 \mathrm{~mm}, \mathrm{~N}$ type $)$ for the control experiments was also supplied by Sigma Aldrich. L-cysteine for the SERS studies was purchased from Merck. All the chemicals were used as received. The stock solutions used in the studies were prepared in ultrapure water (18.2 M $\Omega$-cm) obtained from double stage water purifier (ELGA PURELAB Option-R7). Fresh leaves from the 5 different plants, Gladiolus (Gladiolus dalenii), Indian cress (Tropaeolum majus), Colocasia (Colocasia esculenta), water lily (Nymphaea nauchali) and banana (Musa acuminata) were collected from Indian institute of Technology Mandi, Kamand campus, Himachal Pradesh, India - $\left(31^{\circ} 42^{\prime} 25^{\prime \prime} \mathrm{N}\right.$ $76^{\circ} 55^{\prime} 54$ 'E).

\subsection{Synthesis of Au nanoparticles}

The synthesis of Au nanoparticles was done using a seed mediated procedure according to the literature report [28]. Seed solutions were first prepared by sodium citrate reduction. In this procedure, $25 \mathrm{~mL}$ of ultrapure $\mathrm{H}_{2} \mathrm{O}$ and $250 \mu \mathrm{L}$ of $1 \mathrm{wt} \% \mathrm{HAuCl}_{4}$ was added to a 250 $\mathrm{mL}$ flask and stirred rapidly while boiling. Then $1 \mathrm{~mL}$ of $1 \mathrm{wt} \%$ sodium citrate was added to it to initiate reduction. The growth solution was prepared by adding $100 \mu \mathrm{L}$ of $1 \mathrm{wt} \% \mathrm{HAuCl}_{4}$ solution to $9.8 \mathrm{~mL}$ of ultrapure $\mathrm{H}_{2} \mathrm{O}$. Then $100 \mu \mathrm{L}$ of seed solution was added to this solution and stirred rapidly at room temperature. In the next step, $20 \mu \mathrm{L}$ of a $1 \mathrm{wt} \%$ sodium citrate solution was added, followed by $100 \mu \mathrm{L}$ of $0.03 \mathrm{M}$ hydroquinone. The nanoparticle formation was immediately evidenced by the colour change of the solution from yellow to rusty red.

\subsection{Fabrication of SERS substrates}

The important step in the fabrication of the SERS substrate is the fixation of the plant leaves as fresh leaves 
dehydrate quickly which leads to the change in their surface topography. The fixation of the fresh leaves was done using glutaraldehyde and any water content was replaced by glycerol using literature reports [29].

Two methods were utilized to fabricate SERS substrates. In first method, a thin layer of Au was sputter coated over the fixated leaf substrates and control $\mathrm{Si}$ wafer substrate using Quorum Q150R ES combined sputter coating instrument. The second method used to fabricate the SERS substrates was the drop cast method, where a $100 \mu \mathrm{L}$ suspension of $\mathrm{Au}$ nanoparticle solution was drop casted on to the fixated leaf substrates and on to the control substrate, and allowed to dry under ambient conditions inside a desiccator. For the SERS measurements, $1 \mathrm{mM}$ solution of L-cysteine in deionized water was drop casted on to each of the substrates and allowed to dry under ambient conditions again inside a desiccator.

\subsection{Characterisation}

High resolution transmission electron microscopy (HRTEM) images were obtained using FEI Tecnai G2 200 S-Twin microscope operating at $200 \mathrm{kV}$. Field emission scanning electron microscopy (FESEM) was done using JFEI Nova Nano SEM-450 microscope. Raman spectroscopic measurements for the SERS studies were performed using Horiba LabRAM high resolution UV-VIS-NIR Raman spectrometer instrument equipped with a confocal microscope. The samples were analyzed using a $633 \mathrm{~nm}$ HeNe laser employing a 20X objective lens to achieve an average spot size of $3 \mu \mathrm{m}$ diameter. The integration time for the analysis was $10 \mathrm{~s}$ and the samples were scanned for 5 times under $10 \%$ of total laser power $(17 \mathrm{~mW})$.

\section{Results and discussion}

In this work, we report a strategy for the sensitive detection of biomolecules by SERS technique, employing natural plant leaves deposited with $\mathrm{Au}$ as substrates. As a model biomolecule, the amino acid Lcysteine, whose structure is shown in Figure 1, has been used for our investigations. In our studies, we used five different plant leaves, including gladiolus (Gladiolus dalenii), Indian cress (Tropaeolum majus), colocasia (Colocasia esculenta), water lily (Nymphaea nauchali) and banana (Musa acuminata), that possess different surface topographical features ranging from hierarchical 3D micro/nanostructures to a near-planar surface devoid of intricate surface textures. Prior to the experiments, in order to prevent the leaves from dehydration, which results in the curling and loss in the surface activity, the leaves were fixated with the glutaraldehyde and water content was replaced by the glycerol to make them stable and preserve the surface topography. In this way, the leaves can be made stable for a long duration of time, which makes it usable for practical applications, and no apparent damage happened to the leaves during our observation for a period of over 3 months. Furthermore, the fixation process also helps in directly observing the surface topography of the leaves using microscopic techniques without any damage to the leaves or instrument. The photograph of the plant leaves in their natural habitat along with the SEM images of the fixated leaves is shown in Figure 2. It can be evidenced from the SEM images that the surface topography of each of the plant leaf is different from the other, wherein G. dalenii, $T$. majus and $C$. esculenta show hierarchical micro/nanostructures on the surface, while $N$. nauchali and $M$. acuminata have a relatively planar structure with minimal surface features. In particular, the surface topography of G. dalenii and C. esculenta are somewhat similar (as can be seen from the respective magnified SEM images shown as inserts) possessing intricate hierarchical 3D micro/nanostructures. In addition, they contain micropapillae arrays of nanofolds distributed throughout the surface of the leaves. The surface topography of $T$. majus shows the presence of several short wax tubules leading to a rich surface texture. On the other hand, N. nauchali and M. acuminata show a relatively planar surface topography with micrometer level ridges and panes running adjacent to each other.

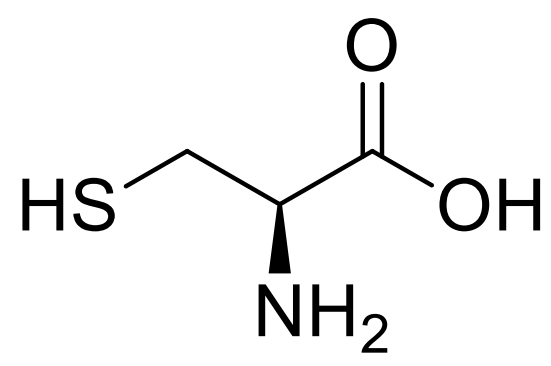

Figure 1. Chemical structure of L-cysteine.

In order to use the fixated plant leaves as substrates for SERS, we had to deposit a plasmonic material on to them. In our work, we used Au for this purpose, as it has been widely reported as a very good material for SERS applications. Specifically, we performed the deposition of $\mathrm{Au}$ on to the fixated plant leaves by two different methods, sputter coating and drop-cast deposition. In sputter coating method, a thin film of Au was deposited on to the fixated leaves, wherein the intrinsic surface topography of the leaves could directly be used to generate electromagnetic hot spots for SERS activity. On the other hand, in drop-cast deposition method, we used separately synthesized Au nanoparticles and deposited them on to the fixated leaf substrates, wherein the nanoparticles gets entrapped within the surface micro/nanostructures in clusters and can serve as electromagnetic hot spots for SERS activity. The TEM image along with the EDAX spectrum of the assynthesized Au nanoparticles used in our work is shown in Figure 3, while Figure 4 shows the SEM images of the $\mathrm{Au}$ deposited fixated plant leaves. 

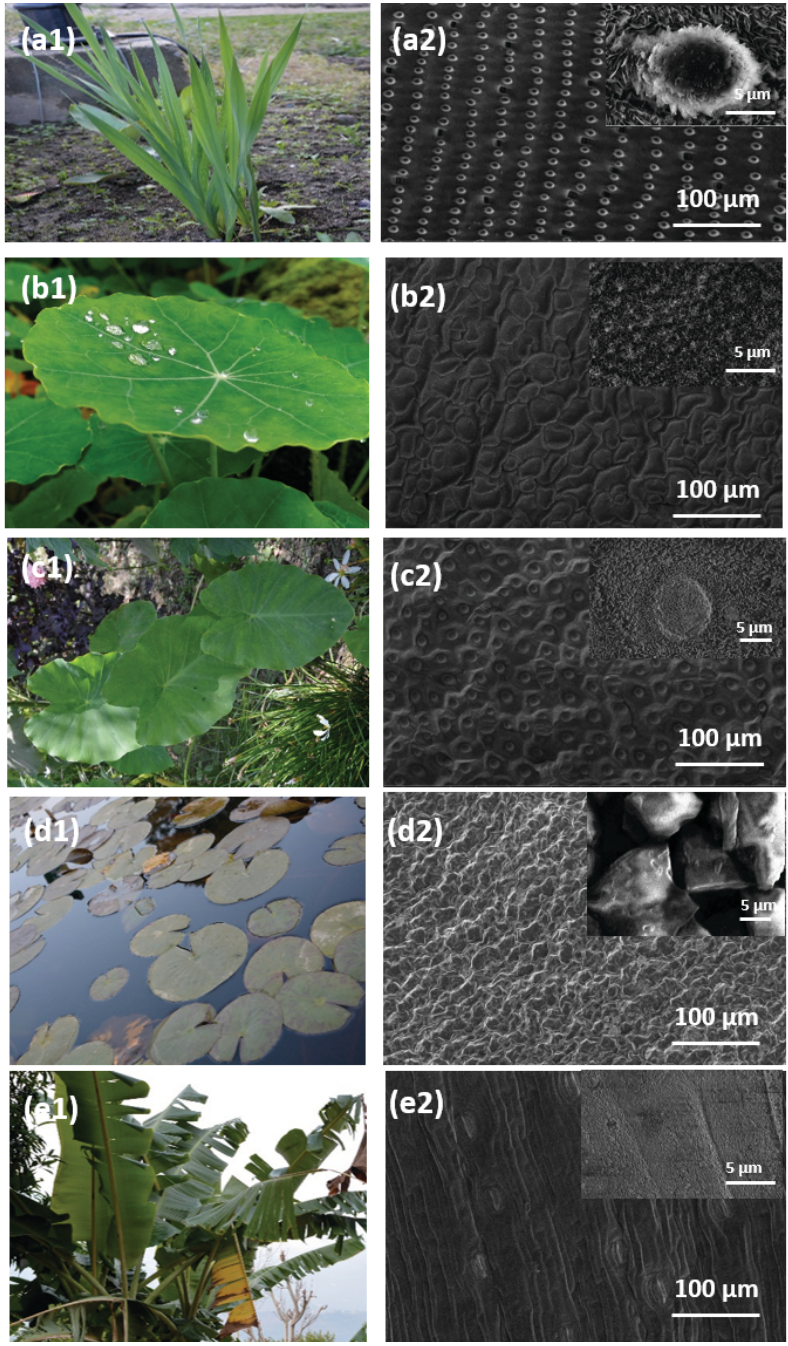

(e2)

Figure 2. Photographs of the plants in their natural habitat (1) and the corresponding SEM images of the fixated leaves (2) of (a) G. dalenii, (b) T. majus, (c) $C$. esculenta, (d) N. nauchali and (e) M. acuminata. Insert shows the magnified SEM image of the fixated leaves.
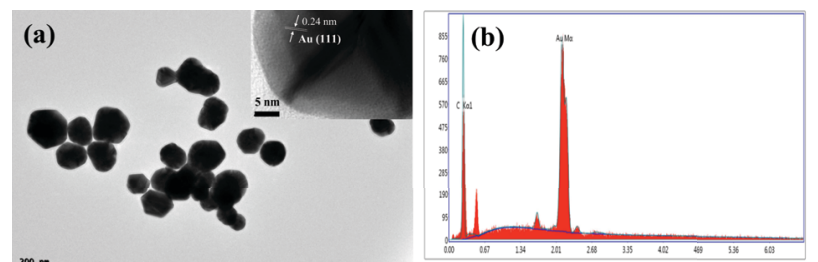

Figure 3. (a) TEM image of Au nanoparticles and (b) its corresponding EDAX spectra.

The above mentioned fixated plant leaves, after $\mathrm{Au}$ deposition, were used as SERS substrates for the sensitive detection of the biomolecular analyte, Lcysteine. The analyte was drop-casted on to each of the substrates in the same volume and concentration, so that the obtained SERS results could be directly compared and correlated with the surface topographical features of the plant leaves. In addition, a smooth $\mathrm{Si}$ wafer, deposited with $\mathrm{Au}$, was used as a control substrate. The SERS spectra of L-cysteine obtained for the sputter coated and drop casted plant leaf based substrates are shown in Figure 5. It can be observed that all the peaks obtained in the spectra correspond to the signature peaks of L-cysteine as reported in literature [30] and no additional peaks, possibly arising from the leaf substrate or background, were evidenced. All the spectra showed a typical peak at $500 \mathrm{~cm}^{-1}$, attributable to the $\mathrm{S}-\mathrm{S}$ stretching vibration in the L-cysteine molecule. Also the characteristic bands of L-cysteine at $619 \mathrm{~cm}^{-1}$ (C-S stretching), $681 \mathrm{~cm}^{-1}$ (C-S stretching), $1201 \mathrm{~cm}^{-1}\left(\mathrm{C}_{\alpha}-\mathrm{H}\right.$ bending) and $1201 \mathrm{~cm}^{-1}\left(\mathrm{CH}_{2}\right.$ twisting $)$ could be evidenced in the spectra. Slight blue shifting of the characteristic peaks was observed, which can be due to the charge transfer interaction between L-cysteine and the Au nanoparticles.
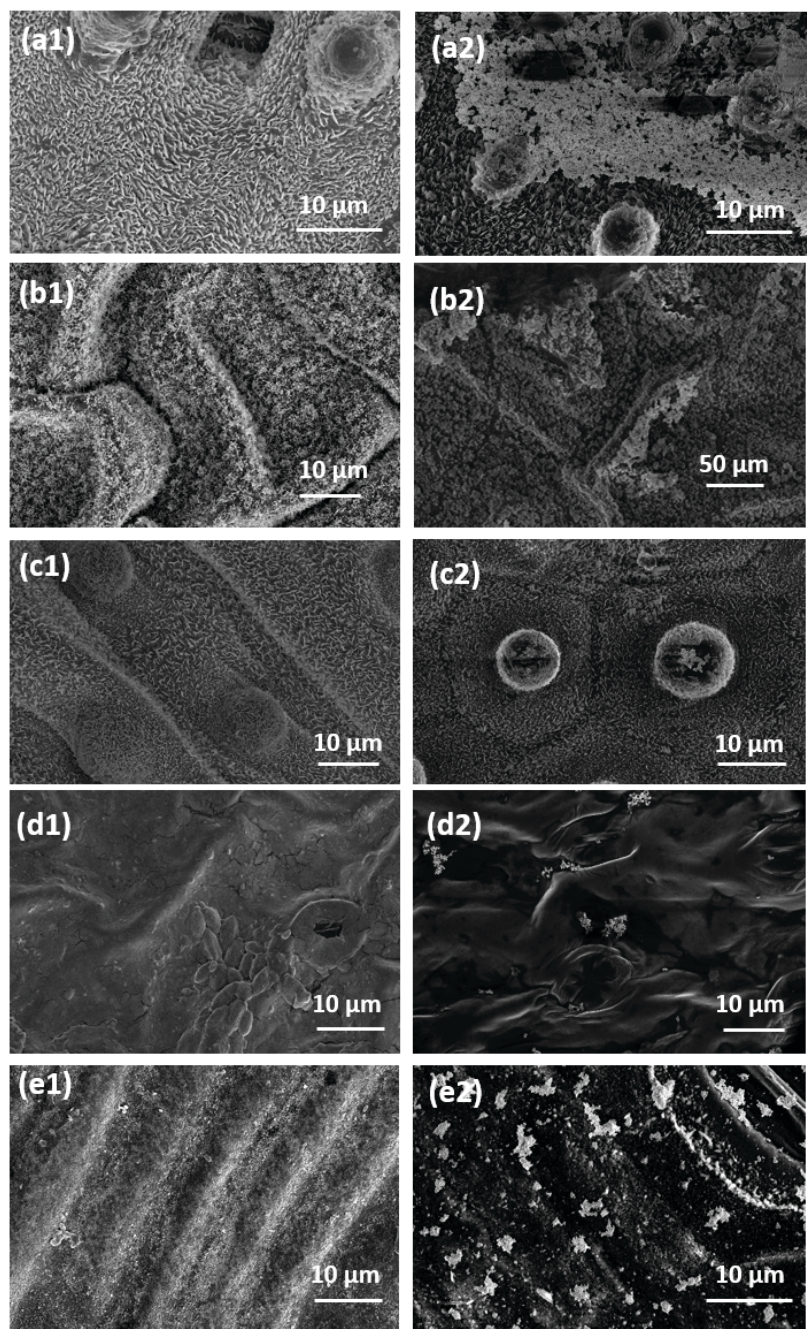

Figure 4. SEM images of Au sputter coated (1) and Au nanoparticles drop casted (2) on fixated plant leaves ((a) G. dalenii, (b) T. majus, (c) C. esculenta, (d) N. nauchali and (e) M. acuminata).

We examined the SERS performance of the $\mathrm{Au}$ deposited leaf surfaces of $G$. dalenii, T. majus, $C$. esculenta, N. nauchali and M. acuminata. All the chosen leaf substrates have different hierarchical structures naturally, which can be related to the enhanced SERS signals from the fabricated substrates. Figure 5 (a) shows the SERS spectra of L-cysteine on sputter coated leaf substrates and Figure 5 (b) shows the variation of SERS 
intensity of the peak at $500 \mathrm{~cm}^{-1}$ for each of the plant leaves. As seen clearly in the spectra, all the leaf substrates show significant enhancement in the signals as compared to the smooth Si wafer (control substrate). In addition, there is a significant difference between the performances of the SERS substrates based on different leaves. The SERS performance based upon the intensity of the signals obtained from the different substrates followed order: G. dalenii $>M$. acuminata $>N$. nauchali $>C$. esculenta $>T$. majus. Distinguished based on the contrast difference between the SEM images (Figure 4) it can be seen that the Au layer covered the whole surface including 3D hierarchical structures, in all the cases. Among all the fabricated substrates, G. dalenii leaves provide the best results wherein the papilla apex is present in ogive-like structure containing some sharp edges and sub-micron wax tubules. The other leaves with intricate hierarchical micro/nanostructures, $C$. esculenta and $T$. majus containing the sub-micrometer wax tubules but without the well-defined ogive-like structures, also show good enhancement but lesser than the $G$. dalenii. For the enhancement in the signals in these cases, we propose that the presence of ogive-like structures with sharp edges and the sub-micron tubular structures are responsible. These structures causes increase in the electric field intensity and subsequently the enhanced SERS performance. Notably in the $M$. acuminata, where there are well defined ridges and panes, arranged almost in parallel, with an average width of 5 microns extend from the bottom to the top, are responsible for the increase of electric field intensity and the formation of electromagnetic hot spots, contributing to SERS enhancement. Similarly in the case of $N$. nauchali, the micrometer level folds and the micrometer level 3D folding is responsible for the enhancement.
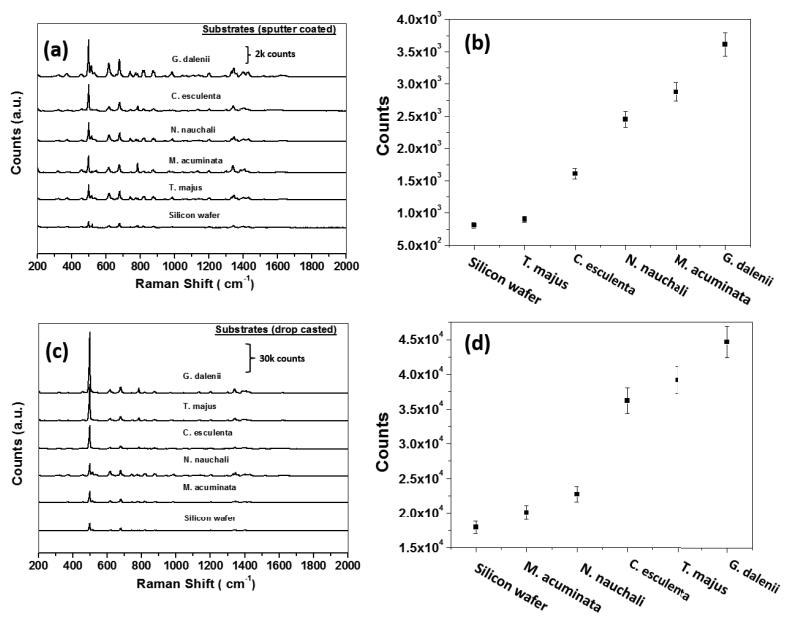

Figure 5. SERS spectra of L-cysteine for the different leaf substrates: (a) sputter coated and (c) drop casted; (b) and (d) show the variation of SERS intensity of the peak at $500 \mathrm{~cm}^{-1}$, for sputter coated and drop casted plant leaf based substrates, respectively shown along with the smooth Si wafer, used as control.

Figure 5 (c) shows the SERS spectra of all the fabricated leaf substrates along with the control $\mathrm{Si}$ substrate drop casted with the $\mathrm{Au}$ nanoparticles and
Figure 5 (d) shows the variation of SERS intensity of the peak at $500 \mathrm{~cm}^{-1}$ for each of the plant leaves. Strong enhancement in the Raman signal is clearly evident due to the presence of the $\mathrm{Au}$ nanoparticle clusters on the fixated plant leaf based substrates. As observed from the spectra acquired from the drop casted SERS substrates, the leaves possessing intricate hierarchical 3D micro/nanostructures on their surface $(G$. dalenii, $T$. majus, and C. esculenta,) show better enhancement of the signals as compared to those with relatively planar surfaces $(N$. nauchali and $M$. acuminata). The intricate micro/nanostructures found on G. dalenii, T. majus, and C. esculenta lead to the entrapment of larger number of nanoparticles to form nanoclusters and thereby enhance the electric field intensity of the electromagnetic hot spots, which is responsible for increase in the Raman signal intensities of the analyte molecule. In this case as well, the Au nanoparticle decorated G. dalenii leaves provided the best enhancements in SERS signals, followed by the C.esculenta, T. majus, N. nauchali and $M$. acuminata. Based on our results, we can conclude that the three dimensional distribution of the $\mathrm{Au}$ nanoparticle clusters formed on the ogive-like protrusions on the surface of $G$. dalenii was better than the 3D arrangement of $\mathrm{Au}$ nanoparticle clusters on the micro/nanostructures present on the surfaces of $T$. majus and C. esculenta. The relatively low SERS enhancement on $N$. nauchali and $M$. acuminata could be attributed to their near-planar surface texture, which leads to the dispersion of $\mathrm{Au}$ nanoparticles and subsequently the formed nanoclusters are small comprising of fewer numbers of nanoparticles. In both sputter coated and drop casted cases, the smooth Si wafer, which is devoid of any micro/nanostructures on its surface had the least SERS performance in the detection of L-cysteine.

The enhancement factor (EF) for the plant leaf based SERS substrates was calculated by the following equation [31]:

$$
E F=\frac{I_{S E R S} / C_{S E R S}}{I_{R S} / C_{R S}}
$$

where, $\mathrm{I}_{\mathrm{SERS}}$ is the intensity of enhanced spectrum, $\mathrm{C}_{\mathrm{SERS}}$ is the concentration of analyte in enhanced spectrum, $I_{R S}$ is the intensity of the Raman spectra of L-cysteine alone and $C_{R S}$ is the concentration of L-cysteine without any substrate. The EF values determined for the different substrates are shown in Figure 6.

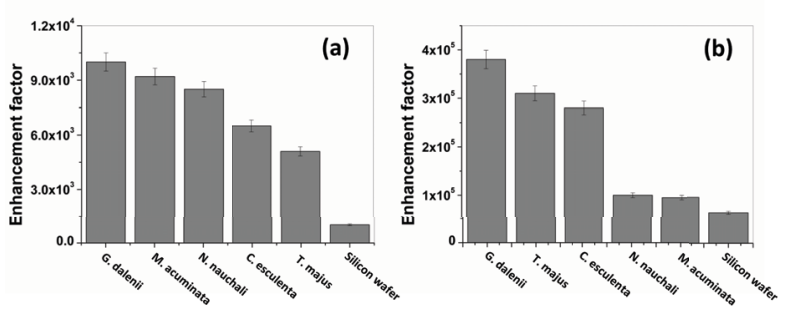

Figure 6. Enhancement factors for (a) sputter coated and (b) drop casted substrates plant leaf based SERS substrates along with the smooth Si wafer used a control. 
The enhancement factors reported in this work correlate well with the corresponding values reported in literature for similar systems such as butterfly wings, plant leaves, flower petals, and other biological surfaces used for SERS applications [23, 25, 32]. In addition, the $\mathrm{Au}$ deposited fixated leaf substrates had reproducible SERS signals.

\section{Conclusions}

In this work, we have demonstrated the sensitive detection of L-cysteine (a model biomolecule) using SERS technique by employing natural plant leaves as facile, low cost and eco-friendly substrates. In particular, we have reported on the influence of the surface topography of five different plant leaf based substrates (deposited with $\mathrm{Au}$ ) on the SERS performance. Furthermore, we have also compared the effect of sputter coated $\mathrm{Au}$ thin films with drop casted Au nanoparticles on the leaf substrates. Based on our results, we claim that the leaves possessing intricate hierarchical 3D micro/nanostructures show higher SERS enhancement compared to the leaves having a near-planar surface. In addition, leaves having drop-casted Au nanoparticle clusters performed better than the leaves sputter coated with a thin Au film.

\section{Acknowledgements}

We are thankful to Advanced Materials Research Center (AMRC), IIT Mandi for laboratory and the characterization facilities. VK acknowledges the financial support from DST, India under INSPIRE Faculty Award. VS acknowledges scholarship from MHRD, India.

\section{References}

1 M. Karplus and J. A. McCammon, Nature Structural \& Molecular Biology 9 (9), 646 (2002).

2 P. Kask, K. Palo, D. Ullmann, and K. Gall, Proceedings of the National Academy of Sciences 96 (24), 13756 (1999).

3 P. Stepnicka, Ferrocenes: ligands, materials and biomolecules. (John Wiley \& Sons, 2008).

4 C. A. Heid, J. Stevens, K. J. Livak, and P. M. Williams, Genome research 6 (10), 986 (1996);

P. V. Hornbeck, Current protocols in immunology, 2.1. 1 (1991).

5 C. Herrmann and M. Reiher, in Atomistic Approaches in Modern Biology (Springer, 2006), pp. 85; S. Song, Y. Qin, Y. He, Q. Huang, C. Fan, and H.-Y. Chen, Chemical Society Reviews 39 (11), 4234 (2010).

6 G. E. Schulz and R. H. Schirmer, Principles of protein structure. (Springer Science \& Business Media, 2013).
I. Nabiev, G. Chumanov, and R. Efremov, Journal of Raman spectroscopy 21 (1), 49 (1990).

D. A. Long, New York, 1 (1977).

N. Colthup, Introduction to infrared and Raman spectroscopy. (Elsevier, 2012).

B. Sharma, R. R. Frontiera, A.-I. Henry, E. Ringe, and R. P. Van Duyne, Materials today 15 (1), 16 (2012).

B. Sharma, M. F. Cardinal, S. L. Kleinman, N. G. Greeneltch, R. R. Frontiera, M. G. Blaber, G. C. Schatz, and R. P. Van Duyne, MRS bulletin 38 (08), 615 (2013).

M. Fleischmann, P. J. Hendra, and A. McQuillan, Chemical Physics Letters 26 (2), 163 (1974); D. L. Jeanmaire and R. P. Van Duyne, Journal of Electroanalytical Chemistry and Interfacial Electrochemistry 84 (1), 1 (1977).

A. Campion and P. Kambhampati, Chemical Society Reviews 27 (4), 241 (1998).

H. Ko and V. V. Tsukruk, small 4 (11), 1980 (2008); L. Zhang, X. Gong, Y. Bao, Y. Zhao, M. Xi, C. Jiang, and H. Fong, Langmuir 28 (40), 14433 (2012).

P. L. Stiles, J. A. Dieringer, N. C. Shah, and R. P. Van Duyne, Annu. Rev. Anal. Chem. 1, 601 (2008).

D. Cialla, A. März, R. Böhme, F. Theil, K. Weber, M. Schmitt, and J. Popp, Analytical and bioanalytical chemistry 403 (1), 27 (2012).

A. Tao, F. Kim, C. Hess, J. Goldberger, R. He, Y. Sun, Y. Xia, and P. Yang, Nano letters 3 (9), 1229 (2003).

R. A. Tripp, R. A. Dluhy, and Y. Zhao, Nano Today 3 (3), 31 (2008).

G. L. Liu and L. P. Lee, Applied Physics Letters 87 (7), 074101 (2005).

P. Rajapandiyan and J. Yang, Journal of Raman Spectroscopy 45 (7), 574 (2014).

C. H. Lee, M. E. Hankus, L. Tian, P. M. Pellegrino, and S. Singamaneni, Analytical chemistry 83 (23), 8953 (2011).

V. Sharma, S. Kumar, K. L. Reddy, A. Bahuguna, and V. Krishnan, Journal of Molecular and Engineering Materials 4 (01), 1640006 (2016); V. Sharma, M. Sharma, S. Kumar, and V. Krishnan, Flora-Morphology, Distribution, Functional Ecology of Plants 224, 59 (2016).

N. L. Garrett, P. Vukusic, F. Ogrin, E. Sirotkin, C. P. Winlove, and J. Moger, Journal of biophotonics 2 (3), 157 (2009).

J.-A. Huang, Y.-L. Zhang, Y. Zhao, X.-L. Zhang, M.-L. Sun, and W. Zhang, Nanoscale 8 (22), 11487 (2016); S.-Y. Chou, C.-C. Yu, Y.-T. Yen, K.-T. Lin, H.-L. Chen, and W.-F. Su, Analytical chemistry 87 (12), 6017 (2015).

V. Sharma, S. Kumar, A. Jaiswal, and V. Krishnan, ChemistrySelect (2017), DOI: 10.1002/slct.201601451 
26 H. Ko, S. Singamaneni, and V. V. Tsukruk, Small 4 (10), 1576 (2008).

27 M. Chen, I. Y. Phang, M. R. Lee, J. K. W. Yang, and X. Y. Ling, Langmuir 29 (23), 7061 (2013).

28 S. D. Perrault and W. C. Chan, Journal of the American Chemical Society 131 (47), 17042 (2009).

29 H. Ensikat, P. Ditsche-Kuru, W. Barthlott, and A. Méndez-Vilas, Microscopy: Science, technology, applications and education 1 (13), 248 (2010); H. Ensikat and W. Barthlott, Journal of microscopy 172 (3), 195 (1993).

H. Lee, M. S. Kim, and S. W. Suh, Journal of Raman spectroscopy 22 (2), 91 (1991).

31 V. Sharma, N. Sinha, S. Dutt, M. Chawla, and P. F. Siril, Journal of colloid and interface science 463, 180 (2016).

32 Z. Mu, X. Zhao, Z. Xie, Y. Zhao, Q. Zhong, L. Bo, and Z. Gu, Journal of Materials Chemistry B 1 (11), 1607 (2013); X. Liu, C. Zong, K. Ai, W. He, and L. Lu, ACS applied materials \& interfaces 4 (12), 6599 (2012). 